\title{
Original article \\ Cultivable bacterial diversity and amylase production in three typical Daqus of Chinese spirits
}

\author{
Zuming Li, ${ }^{1,2}$ Zhihui Bai, ${ }^{2 *}$ Deliang Wang, ${ }^{3}$ Wujiu Zhang, ${ }^{3}$ Michael Zhang, ${ }^{4}$ Francis Lin, ${ }^{4}$ Liping Gao, ${ }^{1}$ \\ Bodi Hui ${ }^{1}$ \& Hongxun Zhang $^{2}$ \\ 1 College of Arts and Sciences of Beijing Union University, Beijing 100191, China \\ 2 Research Center for Eco-Environmental Sciences, Chinese Academy of Sciences, Beijing 100085, China \\ 3 China National Research Institute of Food and Fermentation Industries, Beijing 100027, China \\ 4 Department of Physics and Astronomy, University of Manitoba, Winnipeg, MB R3T 2N2, Canada
}

(Received 22 June 2013; Accepted in revised form 28 August 2013)

\begin{abstract}
Summary Both culture-dependent method and molecular technique were firstly used to simultaneously investigate the cultivable bacterial diversity and amylase production in three typical Daqus of Chinese spirits. The results showed that both cultivable bacterial diversity and amylase production were obviously different. The species of nine bacteria from Deshan, nine from Baisha and six from Wuling Daqus were identified. The total bacterial strains of 17,15 and 14 , and 9,16 and 10 could produce $\alpha$-amylase and glucoamylase, respectively, from the Daqus, and the enzyme yields were different. Bacillus licheniformis, Bacillus subtilis and Bacillus amyloliquefaciens not only were dominant bacteria in the Daqus, but also possessed high activities of $\alpha$-amylase and glucoamylase. By comparison, Bacillus cereus and Bacillus oleronius were found to be another predominant bacterial species and good producers of $\alpha$-amylase and glucoamylase in Deshan and Wuling Daqus, respectively.
\end{abstract}

Keywords $16 \mathrm{~S}$ rRNA gene, bacterial diversity, Daqu, glucoamylase, $\alpha$-amylase.

\section{Introduction}

Chinese spirits are commonly known as 'Chinese liquor', which is one of the six most well-known distilled spirits in the world (Wang et al., 2011). The total annual production of Chinese spirits was estimated at approximately 5 million metric tons per year, and the reason of the popularity of Chinese spirits is the diversity of flavours (Zheng et al., 2012). According to the flavours, Chinese spirits can be roughly classified into strong flavour, light flavour, sauce flavour and strong sauce flavour (Shi et al., 2009; Liu et al., 2012). The spirits are made from cereals, mainly sorghum, by fermentation, distillation and maturation. Mixed culture starters are locally called 'Daqu'. Daqu, which is made from raw wheat, barley and/or pea, is used as inoculum for the fermentation of Chinese spirits (Zheng et al., 2012).

Daqu is an important saccharifying and fermenting agent for the production of Chinese spirits, associated with the degradation of starch to produce alcohol, and also is a determinative factor for the flavouring of

*Correspondent: Fax: +86 1062923563 ;

e-mail: zhbai@rcees.ac.cn spirits (Zheng et al., 2011; Liu et al., 2012; Zheng et al., 2012). During Daqu-making process, the raw materials are typically milled, mixed with water, pressed into a brick-shaped block and then incubated under nonsterile conditions (Zhang et al., 2011). Daqu contains microbes and enzymes, and both glucoamylase and $\alpha$-amylase are two important amylases for starch hydrolysis (Yoshizaki et al., 2010; Qian et al., 2011). In brewing industry, the hydrolysates are used as nutrients in microbial fermentation for ethanol production after enzymatic hydrolysis (Dhale \& VijayRaj, 2009). However, amylase production of bacteria from Daqus is poorly understood. During fermentation, degradation of biopolymers such as starch and formation of aromatic compounds are usually done by microbes (Wang et al., 2008). Therefore, it is important to check the exact composition of the natural product microflora, characterise and distinguish them at a species level (Chen et al., 2011). However, very little is known about the specific microbes associated with Daqus, despite the fact that the subject has been studied by some microbiologists (Li et al., 2011).

Traditional microbiological methods, such as isolation and enumeration in selective media, are effective 
in qualitative and quantitative analysis (Wang et al., 2008; Shi et al., 2009). The microorganisms were isolated from Daqus and then physiological and morphological tests are typically employed for identification. However, phenotypic tests required prolonged periods, and microbial phenotypic characteristics are often negatively affected by culture conditions. Those inaccurate descriptions of characteristics may lead to the misidentification of microbial species (Liu et al., 2012). Molecular methods provide a precise insight into microbial diversity and allow a rapid, high-resolution description of microbial communities by targeting ribosomal genes, which have been used to distinguish or identify the bacteria and fungi associated with Chinese spirits and other foods (Shi et al., 2009; Li et al., 2011; Liu et al., 2012; Zheng et al., 2012). 16S rRNA gene sequence analysis is commonly used for identifying bacteria and constructing bacterial phylogenetic relationships (Huang et al., 2012). PCR-based molecular marker methods have widely been applied in many fields such as in food, agriculture and medicine. In food processing and food preservation, the PCR is a very good tool for quality assurance and food microbe detection (Minh \& Rakshit, 2009). For example, the microbial diversity of Irish edible brown seaweeds had been investigated by combined culture-dependent method and 16S rRNA molecular technique (Gupta et al., 2010). The microbial population densities and speciation of bacterial communities in dried dairy dietary supplements had been investigated using serial dilution plating methods and 16S rRNA molecular techniques, respectively (Hayes et al., 2012). In this study, we firstly report an investigation of the cultivable bacterial diversity and amylase production from three typical Daqus of Chinese spirits.

\section{Materials and methods}

\section{Daqu samples}

The three Daqus were obtained from three main factories of Chinese spirits in Hunan province, and they are famous Chinese spirits in China. The sampling data are shown in Table 1. Three parallel samples were collected from the top, middle and bottom of the store room. After milling and mixing, samples were then transferred to sterile bags, sealed and stored at $4{ }^{\circ} \mathrm{C}$ for further use.

\section{Strain isolation and screening}

Serial dilution plating method was used for the isolation. Ten grams of Daqu powder was homogenised in $90 \mathrm{~mL}$ of sterile $0.9 \% \mathrm{NaCl}$ solution in a rocking incubator for $30 \mathrm{~min}$ at $180 \mathrm{rev} \mathrm{min}^{-1}$. The homogenised samples $(1.0 \mathrm{~mL})$ were serially diluted 10 -fold in sterile $0.9 \% \mathrm{NaCl}$ solution, from which aliquots
Table 1 Samples of three typical Daqus of Chinese spirits

\begin{tabular}{|c|c|c|c|c|}
\hline $\begin{array}{l}\text { Sample } \\
\text { no. }\end{array}$ & $\begin{array}{l}\text { Name } \\
\text { of Daqu }\end{array}$ & Flavour type & $\begin{array}{l}\text { Highest } \\
\text { temperature } \\
\text { inside the } \\
\text { Daqu pile } \\
\left({ }^{\circ} \mathrm{C}\right)\end{array}$ & $\begin{array}{l}\text { Factory and Region } \\
\text { (province, city) }\end{array}$ \\
\hline w & Wuling & Sauce flavour & 65 & $\begin{array}{l}\text { Hunan Wuling } \\
\text { Spirits Co., Ltd., } \\
\text { Hunan, Changde }\end{array}$ \\
\hline B & Baisha & $\begin{array}{l}\text { Strong sauce } \\
\text { flavour }\end{array}$ & 60 & $\begin{array}{l}\text { Changsha Baisha } \\
\text { Spirits Co., Ltd., } \\
\text { Hunan, Changsha }\end{array}$ \\
\hline D & Deshan & Strong flavour & 55 & $\begin{array}{l}\text { Hunan Deshan } \\
\text { Wine Industry } \\
\text { Co., Ltd., Hunan, } \\
\text { Changde }\end{array}$ \\
\hline
\end{tabular}

$(0.1 \mathrm{~mL})$ were spread on beef extract peptone agar plates containing $(\mathrm{w} / \mathrm{v}) \quad 0.5 \%$ beef extract, $1.0 \%$ peptone and $0.5 \% \mathrm{NaCl}$. Inoculated plates were incubated at $37^{\circ} \mathrm{C}$ for $24-48 \mathrm{~h}$ for the isolation and screening on the basis of different colony morphologies (diameter, shape, colour, surface and spore).

\section{DNA extraction and amplification}

A previously published extraction method was used to extract the genomic DNA of the isolates ( $\mathrm{Li}$ et al., 2005). The genes coding for bacterial 16S rRNA (16S rDNA) were amplified from the genomic DNA of the isolates with the primer pair $27 \mathrm{f}(20 \mathrm{bp})\left(5^{\prime}\right.$-AGAG TTTGATCMTGGCTCAG, $\quad \mathrm{M}=\mathrm{C}: \mathrm{A})$ and $1492 \mathrm{r}$ (22 bp) (5'- TACGGYTACCTTGTTACGACTT, $\mathrm{Y}=\mathrm{C}: \mathrm{T})$. These are standard primers appropriate for most eubacteria, and the product represents $>97 \%$ of the 16S rDNA sequence (Escherichia coli standard). The amplified product of the appropriate size was about $1.5 \mathrm{kbp}$. PCR cycling was performed with an Applied Biosystems Gene Amp PCR system 2700. PCR amplification reaction conditions were as previously described (Li et al., 2005, 2012).

\section{DNA sequencing and nucleotide accession number}

The amplified product of the appropriate size $(1.5 \mathrm{kbp})$ was sequenced by BGI LifeTech Co., Ltd., Beijing, China. 16S rRNA gene sequence data of the isolates reported in this article have been submitted to GenBank nucleotide sequence databases with the accession numbers.

\section{Amylase production}

The isolates from the Daqus were diluted in sterile $0.9 \% \mathrm{NaCl}$ solution and then plated onto starch agar 
plates containing 2.0\% soluble starch, $1.0 \%$ peptone and $0.5 \% \mathrm{NaCl}$. Inoculated plates were incubated for 1-3 days at $37{ }^{\circ} \mathrm{C}$ to obtain colonial growth. The incubated plates were stained with $0.3 \%$ iodine solution, and the colonies with clear zones formed by the hydrolysis of starch were evaluated as amylase producers.

The medium used for liquid inoculum culture contained $(\mathrm{w} / \mathrm{v})$ beef extract $0.3 \%$, peptone $1.0 \%, \mathrm{NaCl}$ $0.5 \%, \mathrm{pH} 7.2$. The medium was sterilised by autoclaving at $121^{\circ} \mathrm{C}$ for $20 \mathrm{~min}$. Medium $(20 \mathrm{~mL})$ in the $150-\mathrm{mL}$ Erlenmeyer flasks was inoculated and incubated at $37{ }^{\circ} \mathrm{C}$ by shaking at $180 \mathrm{rev} \min ^{-1}$ for $24 \mathrm{~h}$.

The medium in a 500-mL Erlenmeyer flask contained $(\mathrm{w} / \mathrm{v})$ soluble starch $2.0 \%$, beef extract $0.5 \%$, peptone $1.0 \%$ and $\mathrm{NaCl} 0.5 \%, \mathrm{pH} 7.2$. After sterilisation, the medium $(100 \mathrm{~mL})$ was inoculated with $6 \mathrm{~mL}$ of a 24-hold inoculum culture, incubated at $37{ }^{\circ} \mathrm{C}$ by shaking at $180 \mathrm{rev} \mathrm{min}^{-1}$ for $48 \mathrm{~h}$, and then was centrifuged at $8000 \mathrm{rev} \min ^{-1}$ at $4{ }^{\circ} \mathrm{C}$ for $15 \mathrm{~min}$ to remove the cells and debris. The supernatant was designated as the crude enzyme for future enzyme activity assays.

\section{Enzyme assays}

For $\alpha$-amylase assays, the starch-iodine method was used (Xie et al., 2007; Shankar et al., 2009). The crude enzyme $(0.1 \mathrm{~mL})$ was added to $4.0 \mathrm{~mL}$ of $0.2 \mathrm{M}$ $\mathrm{Na}_{2} \mathrm{HPO}_{4} / \mathrm{C}_{6} \mathrm{H}_{8} \mathrm{O}_{7} \cdot \mathrm{H}_{2} \mathrm{O}$ buffer solution ( $\mathrm{pH} \quad 6.0$ ) containing $1.0 \%$ soluble starch at $60{ }^{\circ} \mathrm{C}$. After $30 \mathrm{~min}$ of enzyme reaction, $10 \mathrm{~mL}$ of $0.5 \mathrm{~m}$ HAc was added to the solution. Then $1 \mathrm{~mL}$ of the enzyme reaction mixture was added to $10 \mathrm{~mL}$ of iodine solution. Absorbance was measured at $620 \mathrm{~nm}$ using spectrophotometer (WFZ UV-2000; Unico [Shanghai] Instrument Co., Ltd, Shanghai, China). One unit of $\alpha$-amylase activity was defined as the amount of enzyme that hydrolysed $1 \mathrm{mg}$ of starch per hour under the assay conditions. The experiments were run in triplicate, and all standard deviations were under 5\%.

Glucoamylase was determined by measuring released reducing sugars using DNS (dinitrosalicylic acid) method (Smith \& Zahnley, 2005; Kumar \& Satyanarayana, 2009). Activity of glucoamylase was evaluated using $1.0 \%$ of starch solution in $0.2 \mathrm{M} \mathrm{Na} \mathrm{NaPO}_{4} /$ $\mathrm{C}_{6} \mathrm{H}_{8} \mathrm{O}_{7} \cdot \mathrm{H}_{2} \mathrm{O}$ buffer ( $\mathrm{pH}$ 6.0) as substrate. A known volume of $0.5 \mathrm{~mL}$ of crude enzyme was added to $1.5 \mathrm{~mL}$ of substrate solution that had been pre-incubated at $40{ }^{\circ} \mathrm{C}$ for $5 \mathrm{~min}$. The enzyme-starch mixture was incubated for $30 \mathrm{~min}$ at $40{ }^{\circ} \mathrm{C}$ and then $1.5 \mathrm{~mL}$ of DNS reagent was added. Samples were boiled for $15 \mathrm{~min}$, cooled to room temperature and diluted with $21.5 \mathrm{~mL} \mathrm{H}_{2} \mathrm{O}$. The absorbance of the samples at $540 \mathrm{~nm}$ was determined using spectrophotometer (WFZ UV-2000, Unico [Shanghai] Instrument Co., Ltd). One unit of glucoamylase activity was defined as the amount of enzyme that liberated $1 \mathrm{mg}$ of reducing sugar as glucose per hour under the assay conditions. The experiments were run in triplicate, and all standard deviations were under $5 \%$.

\section{Results and discussion}

\section{Bacterial diversity}

A total of thirty-four, twenty-four and fifty-six bacterial isolates were randomly screened and identified by sequencing the $16 \mathrm{~S}$ rDNA from Deshan, Baisha and Wuling Daqu samples, respectively. The sequences had been submitted to the GenBank database with accession numbers (Tables 2-4). For the three bacteria, the sequences fell into nine, nine and six species. Bacillus genus was predominant in the Daqus. Indeed, $85.3 \%$ of the sequences identified from Deshan Daqu belonged to the species of $B$. licheniformis, B. subtilis, $B$. cereus and $B$. amyloliquefaciens, while $70.8 \%$ of the sequences identified from Baisha Daqu belonged to the species of B. licheniformis, B. subtilis and B. amyloliquefaciens. Other bacteria such as B. megaterium, Geobacillus stearothermophilus, B. altitudinis, Brevibacillus parabrevis and $B$. anthracis were encountered in Deshan Daqu. Meanwhile, B. cereus, B. fordii, B. flexus, B. methylotrophicus, Staphylococcus kloosii and $B$. sonorensis were found in Baisha Daqu. In contrast, $94.6 \%$ of the sequences were identified from Wuling Daqu, which belonged to the species B. licheniformis, B. subtilis, B. amyloliquefaciens and B. oleronius. Other bacteria such as $B$. circulans and $B$. sonorensis were observed in Wuling Daqu.

To understand the phylogenetic position of these strains from the typical Daqus, we constructed phylogenetic trees based on the comparison of $16 \mathrm{~S}$ rDNA sequences of the strains from the Daqus and those of reference bacteria (Figs 1a,b, 2 and 3a,b).

Daqu is a mixture of grains, microorganisms, enzymes and aroma precursors. Using Daqu as a starter culture is the unique characteristic of the Chinese spirits. The specific metabolic characteristics of the microorganisms in Daqus can greatly affect the yield and quality of the Chinese spirits; for example, $\alpha$-amylase and glucoamylase produced from microorganisms in Daqus play a key role in starch degradation for supplying fermentable sugars for the fermentation process of the Chinese spirits. Owing to differences in the climate of the brewing geographical origins, production techniques, fermentation process, etc., bacterial diversity, amylase production and Daqu intensity of flavours between the three Daqus were obviously different (Qin et al., 2012).

Bacillus species were the most frequently isolated bacteria from Deshan, Baisha and Wuling Daqus (Figs 1 including a and b, 2 and 3 including $a$ and b). This observation is consistent with those of previous studies (Wang et al., 2008; Shi et al., 2009; Zheng 
Table 2 Bacterial diversity and amylase production in Deshan Daqu

\begin{tabular}{|c|c|c|c|c|c|c|}
\hline $\begin{array}{l}\text { Strain } \\
\text { no. }\end{array}$ & $\begin{array}{l}\text { Related } \\
\text { GenBank } \\
\text { sequence }\end{array}$ & $\begin{array}{l}\text { Closest relative } \\
\text { (NCBI accession no.) }\end{array}$ & Identity $/ \%$ & HC value & $\begin{array}{l}\text { Activities } \\
\text { of } \alpha \text {-amylase } \\
\mathrm{U}^{-1} \mathrm{~mL}^{-1}\end{array}$ & $\begin{array}{l}\text { Activities of } \\
\text { glucoamylase } \mathrm{U}^{-1} \mathrm{~mL}^{-1}\end{array}$ \\
\hline D1 & KC441750 & B. licheniformis HT18 (JN013203) & 100 & - & - & - \\
\hline D2 & KC441751 & B. amyloliquefaciens WAS2-1 (JF496509) & 99.9 & $1.3 \pm 0.1$ & $16.1 \pm 0.6$ & $30.1 \pm 1.3$ \\
\hline D3 & KC441752 & B. subtilis LXA7 (GQ861467) & 99.9 & $1.8 \pm 0.2$ & $5.6 \pm 0.3$ & $28.4 \pm 1.2$ \\
\hline D4 & KC441753 & B. licheniformis LZBL-9 (JX847115) & 100 & - & - & - \\
\hline D5 & KC441754 & B. megaterium SZ-3 (HM061611) & 99.9 & - & - & - \\
\hline D6 & KC441783 & Geobacillus stearothermophilus DDKRC4 (JN641292) & 99.9 & - & - & - \\
\hline D7 & KC441755 & B. licheniformis LZBL-13 (JX847119) & 99.9 & $1.4 \pm 0.1$ & $6.4 \pm 0.3$ & $15.9 \pm 0.7$ \\
\hline D8 & KC441756 & B. amyloliquefaciens SDF004 (JO062537) & 99.9 & - & - & - \\
\hline D13 & KC441757 & B. subtilis LXA7 (GQ861467) & 99.9 & $1.6 \pm 0.2$ & $15.4 \pm 0.5$ & $31.2 \pm 1.3$ \\
\hline D15 & KC441758 & B. amyloliquefaciens Nt1-7 (H0831418) & 99.9 & $2.6 \pm 0.3$ & $36.2 \pm 1.6$ & $43.8 \pm 1.9$ \\
\hline D18 & KC441759 & B. amyloliquefaciens Ht1-1 (JF899275) & 99.9 & $1.9 \pm 0.2$ & $20.4 \pm 0.8$ & $31.8 \pm 1.4$ \\
\hline D19 & KC441760 & B. cereus Cr-50 (JF895490) & 98.9 & - & - & - \\
\hline D20 & KC441761 & B. amyloliquefaciens T004 (H0840415) & 100 & - & - & - \\
\hline D21 & KC441762 & B. cereu CP1 (HO833025) & 99.5 & $1.7 \pm 0.2$ & $14.2 \pm 0.5$ & - \\
\hline D23 & KC441763 & B. subtilis LXB3 (GQ861468) & 99.9 & - & - & - \\
\hline D24 & KC441764 & B. amyloliquefaciens BAB-807 (KC250199) & 100 & $2.1 \pm 0.3$ & $11.9 \pm 0.4$ & $12.3 \pm 0.4$ \\
\hline D26 & KC441765 & B. cereus JY7 (H0833025) & 99.7 & - & - & - \\
\hline D28 & KC441766 & B. subtilis CBZ-2 ( $\mathrm{KC} 142124)$ & 100 & $1.7 \pm 0.2$ & $7.7 \pm 0.3$ & $19.1 \pm 0.8$ \\
\hline D29 & KC441767 & B. subtilis HT19 (JN013204) & 100 & $2.1 \pm 0.3$ & $21.3 \pm 0.8$ & $38.9 \pm 1.7$ \\
\hline D30 & KC441768 & B. cereus KVP109 (JX290089) & 100 & $1.5 \pm 0.1$ & $15.2 \pm 0.5$ & $10.1 \pm 0.4$ \\
\hline D31 & KC441769 & B. subtilis SY-A4 (JN656163) & 100 & - & - & - \\
\hline D34 & KC441770 & B. altitudinis JF31 (KC172006) & 100 & - & - & - \\
\hline D35 & KC441771 & B. subtilis YT2 (HO143571) & 99.9 & - & - & - \\
\hline D36 & KC441772 & Brevibacillus parabrevis NBRC 12335 (AB680275) & 99.9 & $1.7 \pm 0.2$ & $1.9 \pm 0.1$ & - \\
\hline D38 & KC441773 & B. licheniformis BaDB24 (JX237858) & 100 & $1.4 \pm 0.1$ & $5.8 \pm 0.3$ & $64.9 \pm 2.8$ \\
\hline D39 & KC441774 & B. licheniformis SCC112026 (JN998723) & 99.9 & - & - & - \\
\hline D40 & KC441775 & B. anthracis $\mathrm{SH} 123(\mathrm{KC} 172044)$ & 99.9 & $1.7 \pm 0.2$ & $10.3 \pm 0.4$ & $20.1 \pm 0.8$ \\
\hline D41 & KC441776 & B. amyloliquefaciens FJWHG14 (H0853016) & 100 & $2.2 \pm 0.3$ & $26.5 \pm 1.1$ & $57.5 \pm 2.5$ \\
\hline D42 & KC441777 & B. cereus FM10 (DO289077) & 99.4 & - & - & - \\
\hline D43 & KC441778 & B. licheniformis WAS3-5 (JF496512) & 99.9 & $1.5 \pm 0.1$ & $13.3 \pm 0.4$ & $31.3 \pm 1.3$ \\
\hline D46 & KC441779 & B. licheniformis KJN-1 (JO619622) & 99.9 & - & - & - \\
\hline D48 & KC441780 & B. licheniformis ISA7 (HO189751) & 100 & - & - & - \\
\hline D49 & KC441781 & B. cereus CP1 (JX544748) & 98.9 & - & - & - \\
\hline D50 & KC441782 & B. licheniformis NJ-5 (FJ435674) & 100 & $1.2 \pm 0.1$ & $10.7 \pm 0.4$ & $14.6 \pm 0.5$ \\
\hline
\end{tabular}

-, means the value of parameter was not detected.

et al., 2012). The Bacillus dominant in the Daqus was an endospore-producing bacterium, which was able to endure an unfavourable environment, and had a better ability than other bacteria to survive under low moisture content (13-14\%) and high temperature $\left(60-65^{\circ} \mathrm{C}\right)$ (Liu et al., 2012). Bacillus species are important sources of proteases and amylases, and the hydrolytic capabilities of these microorganisms can result in a precursor-rich environment, which is useful for subsequent reactions leading to flavour production (Wang et al., 2008). In addition, Bacillus spp. produce nitrogenous flavour compounds such as diverse pyrazines to contribute to Daqu flavour (Zheng et al., 2012). We noted that B. licheniformis and B. subtilis were the dominant bacteria in the Daqus (Figs 1, 2 and 3 ). This is in accordance with earlier reports (Wang et al., 2008; Shi et al., 2009; Zheng et al., 2012).
Although Deshan, Baisha and Wuling Daqus were all produced in Hunan province, they are strong flavour Daqu, strong sauce flavour Daqu and sauce flavour Daqu, respectively. The cultivable bacterial diversity was obviously different between them. It was observed that the dominant bacteria existing in the Daqus were $B$. licheniformis, B. subtilis and B. amyloliquefacien (Figs 1, 2 and 3). Bacillus amyloliquefaciens was also found in Daqu of Maotai spirit (Liu et al., 2012). We also found that $B$. cereus and $B$. oleronius were another dominant bacterial species in Deshan and Wuling Daqus, respectively (Figs 1 and 3). But B. oleronius was not the dominant bacterial species in Maotai Daqu, which is a sauce flavour Daqu from Guizhou Province (Liu et al., 2012). Other species, such as B. anthracis, B. altitudinis, B. megaterium, Geobacillus stearothermophilus and Brevibacillus parabrevis, were all in Deshan 
Table 3 Bacterial diversity and amylase production in Baisha Daqu

\begin{tabular}{|c|c|c|c|c|c|c|}
\hline $\begin{array}{l}\text { Strain } \\
\text { no. }\end{array}$ & $\begin{array}{l}\text { Related GenBank } \\
\text { sequence }\end{array}$ & Closest relative (NCBI accession no.) & Identity /\% & HC value & $\begin{array}{l}\text { Activities of } \\
\alpha \text {-amylase } \mathrm{U}^{-1} \mathrm{~mL}^{-1}\end{array}$ & $\begin{array}{l}\text { Activities of } \\
\text { glucoamylase } \mathrm{U}^{-1} \mathrm{~mL}^{-1}\end{array}$ \\
\hline B2 & KC441726 & B. licheniformis BaDB24 (JX237858) & 100 & $1.5 \pm 0.1$ & $8.3 \pm 0.3$ & $9.3 \pm 0.3$ \\
\hline B5 & KC441727 & B. licheniformis LZBL-9 (JX847115) & 99.9 & $1.2 \pm 0.1$ & $4.5 \pm 0.2$ & $36.2 \pm 1.6$ \\
\hline B8 & KC441728 & B. licheniformis 5.22 (HE801988) & 100 & $1.6 \pm 0.2$ & $6.8 \pm 0.3$ & - \\
\hline B10 & KC441729 & B. fordii 2-6AIA (FN397482) & 99.9 & - & - & - \\
\hline B11 & KC441730 & B. licheniformis DQgbc4 (GQ470399) & 100 & $1.1 \pm 0.1$ & $10.9 \pm 0.4$ & - \\
\hline B12 & KC441731 & B. licheniformis 30N1-9 (JN366720) & 99.9 & - & - & - \\
\hline B18 & KC441732 & B. flexus WY2 (J0936679) & 99.9 & $1.6 \pm 0.2$ & $4.3 \pm 0.2$ & $10.3 \pm 0.4$ \\
\hline B30 & KC441733 & B. licheniformis HT18 (JN013203) & 100 & - & - & - \\
\hline B32 & KC441734 & B. methylotrophicus JF38 (KC172013) & 100 & - & - & - \\
\hline B39 & KC441735 & B. subtilis Aj080718IA-25 (HQ727971) & 99.7 & $2.3 \pm 0.3$ & $26.5 \pm 1.1$ & $4.5 \pm 0.2$ \\
\hline B40 & KC441736 & B. licheniformis TSM2 (JX847115) & 99.9 & - & - & - \\
\hline B47 & KC441737 & B. amyloliquefaciens KtVC2-2 (KC122196) & 100 & $1.7 \pm 0.2$ & $11.4 \pm 0.4$ & $12.5 \pm 0.4$ \\
\hline B49 & KC441747 & B. licheniformis (JN166722) & 99.7 & $1.8 \pm 0.2$ & $12.6 \pm 0.4$ & $11.9 \pm 0.4$ \\
\hline B51 & KC441749 & B. licheniformis SD2 (HM055611) & 99.6 & - & - & - \\
\hline B53 & KC441738 & B. subtilis FS106 (KC179631) & 100 & $1.9 \pm 0.2$ & $13.4 \pm 0.5$ & $50.1 \pm 2.2$ \\
\hline B54 & KC441739 & B. subtilis VITM6 (JX981919) & 100 & $1.8 \pm 0.2$ & $14.1 \pm 0.5$ & $8.3 \pm 0.3$ \\
\hline B57 & KC441740 & B. amyloliquefaciens BAB-807 (KC250199) & 99.9 & $2.1 \pm 0.3$ & $18.5 \pm 0.7$ & $6.3 \pm 0.3$ \\
\hline B59 & KC441741 & B. subtilis NS04 (JX126865) & 99.9 & $1.2 \pm 0.1$ & $4.6 \pm 0.2$ & - \\
\hline B64 & KC441742 & B. cereus C (JX544747) & 100 & $1.6 \pm 0.2$ & $10.8 \pm 0.4$ & - \\
\hline B68 & KC441743 & B. licheniformis 2J-1 (FJ493045) & 99.9 & $1.2 \pm 0.1$ & $3.5 \pm 0.1$ & - \\
\hline B69 & KC441744 & Staphylococcus kloosii S7-447 (J0660231) & 99.9 & - & - & - \\
\hline B71 & KC441745 & B. licheniformis HT17 (JN013202) & 100 & - & - & - \\
\hline B81 & KC441746 & B. flexus MDLD1 (FJ861081) & 100 & - & - & - \\
\hline B83 & KC441748 & B. sonorensis HT1 (JN013186) & 100 & - & - & - \\
\hline
\end{tabular}

- , means the value of parameter was not detected.

Daqu, while B. fordii, B. flexus, B. methylotrophicus and Staphylococcus kloosii were only observed in Baisha Daqu. Additionally, B. circulans were found only in Wuling Daqu. Although both Maotai Daqu and Wuling Daqu belong to sauce flavour Daqu, the cultivable bacterial diversity between them is obviously different. Nested PCR-DGGE analysis of bacterial DNA from the Maotai Daqu highlighted the presence of various species, for example, B. licheniformis, B. subtilis, B. sonorensis, Bacillus sp., Virgibacillus sp. and Lactobacillus buchneri (Liu et al., 2012). Bacteria in Maotai Daqu, which was revealed by culture-dependent method, consisted of Bacillus, Acetobacter, Lactobacillus and Clostridium, among which Bacillus strains were found to be predominant (Wang et al., 2008). A clear advantage of culturing is that a collection of pure cultures is obtained that can be used for further experimentation (Zheng et al., 2012). It is similar that Bacillus is a dominant bacterial genus between them, while B. subtilis and B. licheniformis are their predominant bacterial species too (Wang et al., 2008; Liu et al., 2012).

\section{Amylase production}

Following staining with iodine solution, the isolates from the three Daqus, which exhibited visible clear zones around the colonies on agar plates with starch as the sole carbon source, were selected as good producers of amylase (Tables 2-4).

A total of seventeen and fifteen bacterial isolates from Deshan Daqu could produce extracellular $\alpha$-amylase and glucoamylase with the maximum yield of 36.2 $\mathrm{U} \mathrm{mL}^{-1}$ by $B$. amyloliquefaciens $\mathrm{D} 15$ and 64.9 $\mathrm{U} \mathrm{mL}^{-1}$ by $B$. licheniformis D38 (Table 2). The activity of $\alpha$-amylase and glucoamylase produced by $B$. licheniformis, B. subtilis, B. amyloliquefaciens and B. cereus accounted for $15.2 \%, 28.2 \%, 20.9 \%$ and $26.1 \%$, and $46.5 \%, 39.0 \%, 12.3 \%$ and $2.2 \%$, respectively. Furthermore, B. anthracis could produce both $\alpha$-amylase and glucoamylase, while Brevibacillus parabrevis could produce merely $\alpha$-amylase.

Fourteen and nine bacteria could be isolated from Baisha Daqu, which could produce $\alpha$-amylase and glucoamylase (Table 3). Bacillus subtilis B39 produced the maximum $\alpha$-amylase yield of $26.5 \mathrm{U} \mathrm{mL}^{-1}$, and the maximum glucoamylase yield of $50.1 \mathrm{U} \mathrm{mL}^{-1}$ was produced by $B$. subtilis B53. The activity of $\alpha$-amylase and glucoamylase produced by $B$. licheniformis, B. subtilis and B. amyloliquefaciens accounted for $31.0 \%, 38.4 \%$ and $39.0 \%$, and $42.1 \%, 19.9 \%$ and $12.6 \%$, respectively. In addition, B. flexus could produce both $\alpha$-amylase and glucoamylase, whereas $B$. cereus could produce merely $\alpha$-amylase. 
Table 4 Bacterial diversity and amylase production in Wuling Daqu

\begin{tabular}{|c|c|c|c|c|c|c|}
\hline $\begin{array}{l}\text { Strain } \\
\text { no. }\end{array}$ & $\begin{array}{l}\text { Related GenBank } \\
\text { sequence }\end{array}$ & Closest relative (NCBI accession no.) & Identity /\% & $\mathrm{HC}$ value & $\begin{array}{l}\text { Activities of } \\
\alpha \text {-amylase } \mathrm{U}^{-1} \mathrm{~mL}^{-1}\end{array}$ & $\begin{array}{l}\text { Activities of } \\
\text { glucoamylase } \mathrm{U}^{-1} \mathrm{~mL}^{-1}\end{array}$ \\
\hline W1 & KC441816 & B. subtilis C8-4 (EU257436) & 99.7 & $2.1 \pm 0.3$ & $20.8 \pm 0.8$ & $12.6 \pm 0.4$ \\
\hline W4 & KC441817 & B. licheniformis HT9 (JN013194) & 100 & $1.7 \pm 0.2$ & $12.9 \pm 0.4$ & $2.8 \pm 0.1$ \\
\hline W5 & KC441818 & B. oleronius 30N3-3 (JN366766) & 98.5 & $1.4 \pm 0.1$ & $8.4 \pm 0.3$ & $20.7 \pm 0.8$ \\
\hline W6 & KC441819 & B. licheniformis DQgbc4 (GQ470399) & 99.9 & - & - & - \\
\hline W7 & KC441820 & B. licheniformis HT9 (JN013194) & 100 & - & - & - \\
\hline W8 & KC441821 & B. licheniformis LZBL-13 (JX847119) & 100 & - & - & - \\
\hline W10 & KC441822 & B. amyloliquefaciens BAB-807 (KC250199) & 99.9 & - & - & - \\
\hline W11 & KC441823 & B. licheniformis LZBL-13 (JX847119) & 100 & $1.2 \pm 0.1$ & $1.4 \pm 0.1$ & $4.5 \pm 0.2$ \\
\hline W12 & KC441824 & B. licheniformis LZBL-13 (JX847119) & 99.9 & - & - & - \\
\hline W13 & KC441825 & B. licheniformis LZBL-13 (JX847119) & 99.9 & - & - & - \\
\hline W14 & KC441826 & B. licheniformis 2J-1 (FJ493045) & 99.9 & - & - & - \\
\hline W15 & KC441827 & B. licheniformis ML1 (H0850703) & 99.6 & - & - & - \\
\hline W16 & KC441828 & B. amyloliquefaciens SWM1 (JN851189) & 99.9 & - & - & - \\
\hline W17 & KC441829 & B. licheniformis HU14 (EF101734) & 99.9 & - & - & - \\
\hline W18 & KC441830 & B. licheniformis LZBL-9 (JX847115) & 100 & $1.5 \pm 0.1$ & $3.2 \pm 0.1$ & $0.9 \pm 0.1$ \\
\hline W19 & KC441831 & B. oleronius ATCC 700005 (NR_043325) & 99.5 & - & - & - \\
\hline W20 & KC441832 & B. licheniformis LZBL-13 (JX847119) & 99.7 & - & - & - \\
\hline W21 & KC441833 & B. oleronius Gx8 (JF833092) & 99.4 & $1.6 \pm 0.2$ & $9.6 \pm 0.4$ & - \\
\hline W22 & KC441834 & B. oleronius ATCC 700005 (NR_043325) & 99.7 & $1.7 \pm 0.2$ & $10.7 \pm 0.4$ & - \\
\hline W23 & KC441835 & B. subtilis 9A (KC196261) & 99.9 & $1.8 \pm 0.2$ & $6.5 \pm 0.3$ & $6.2 \pm 0.3$ \\
\hline W24 & KC441836 & B. amyloliquefaciens BAB-807 (KC250199) & 100 & $1.9 \pm 0.2$ & $8.8 \pm 0.3$ & $3.1 \pm 0.1$ \\
\hline W25 & KC441837 & B. amyloliquefaciens BAB-807 (KC250199) & 99.5 & $2.0 \pm 0.3$ & $14.3 \pm 0.5$ & $30.3 \pm 1.3$ \\
\hline W27 & KC441838 & B. licheniformis HT13 (JN013198) & 99.8 & - & - & - \\
\hline W28 & KC441839 & B. licheniformis LZBL-13 (JX847119) & 99.9 & - & - & - \\
\hline W30 & KC441840 & B. licheniformis LZBL-13 (JX847119) & 99.9 & - & - & - \\
\hline W31 & KC441841 & B. licheniformis KJN-1 (J0619622) & 100 & - & - & - \\
\hline W32 & KC441842 & B. licheniformis LZBL-13 (JX847119) & 100 & - & - & - \\
\hline W33 & KC441843 & B. licheniformis HT13 (JN013198) & 99.9 & - & - & - \\
\hline W34 & KC441844 & B. licheniformis HT13 (JN013198) & 99.9 & - & - & - \\
\hline W35 & KC441845 & B. licheniformis HT9 (JN013194) & 99.9 & $1.3 \pm 0.1$ & $3.6 \pm 0.1$ & - \\
\hline W36 & KC441846 & B. licheniformis 2J-1 (FJ493045) & 99.9 & - & - & - \\
\hline W37 & KC441847 & B. licheniformis LZBL-9 (JX847115) & 99.9 & $1.4 \pm 0.1$ & $2.5 \pm 0.1$ & - \\
\hline W41 & KC441848 & B. oleronius 30N3-6 (JN366730) & 99.7 & - & - & - \\
\hline W42 & KC441849 & B. oleronius 30N3-6 (JN366730) & 99.7 & - & - & - \\
\hline W44 & KC441850 & B. licheniformis LZBL-13 (JX847119) & 99.9 & - & - & - \\
\hline W45 & KC441851 & B. licheniformis LZBL-13 (JX847119) & 99.9 & - & - & - \\
\hline W46 & KC441852 & B. oleronius 30N3-6 (JN366730) & 99.9 & - & - & - \\
\hline W47 & KC441853 & B. circulans C2-37c-4 (JX517212) & 99.9 & - & - & - \\
\hline W48 & KC441854 & B. subtilis FS05 (JO403532) & 99.9 & $1.4 \pm 0.1$ & $13.8 \pm 0.5$ & $0.19 \pm 0.04$ \\
\hline W49 & KC441855 & B. amyloliquefaciens BAB-807 (KC250199) & 99.9 & $1.2 \pm 0.1$ & $6.9 \pm 0.3$ & - \\
\hline W50 & KC441856 & B. subtilis 9A (KC196261) & 99.9 & - & - & - \\
\hline W51 & KC441857 & B. licheniformis BaDB24 (JX237858) & 100 & - & - & - \\
\hline W52 & KC441858 & B. licheniformis F3 (EU256500) & 99.9 & - & - & - \\
\hline W53 & KC441859 & B. sonorensis HT1 (JN013186) & 99.9 & - & - & - \\
\hline W55 & KC441860 & B. licheniformis LZBL-13 (JX847119) & 99.7 & - & - & - \\
\hline W56 & KC441861 & B. licheniformis HT9 (JN013194) & 100 & $1.6 \pm 0.2$ & $4.5 \pm 0.2$ & $2.6 \pm 0.1$ \\
\hline W57 & KC441862 & B. licheniformis LZBL-13 (JX847119) & 99.9 & - & - & - \\
\hline W58 & KC441863 & B. licheniformis HT9 (JN013194) & 99.9 & - & - & - \\
\hline W59 & KC441864 & B. licheniformis 55N2-8 (JN366726) & 100 & - & - & - \\
\hline W60 & KC441865 & B. licheniformis LZBL-9 (JX847115) & 99.9 & - & - & - \\
\hline W61 & KC441866 & B. licheniformis BPRIST039 (JF700489) & 99.9 & $2.2 \pm 0.3$ & $22.1 \pm 0.9$ & - \\
\hline W62 & KC441867 & B. licheniformis LZBL-13 (JX847119) & 99.9 & - & - & - \\
\hline W63 & KC441868 & B. licheniformis (JN166722) & 99.7 & - & - & - \\
\hline W64 & KC441869 & B. licheniformis LZBL-13(JX847119) & 100 & - & - & - \\
\hline W65 & KC441870 & B. licheniformis BaDB24 (JX237858) & 100 & - & - & - \\
\hline W66 & KC441871 & B. sonorensis HT1 (JN013186) & 99.9 & - & - & - \\
\hline
\end{tabular}

- , means the value of parameter was not detected. 
(a)

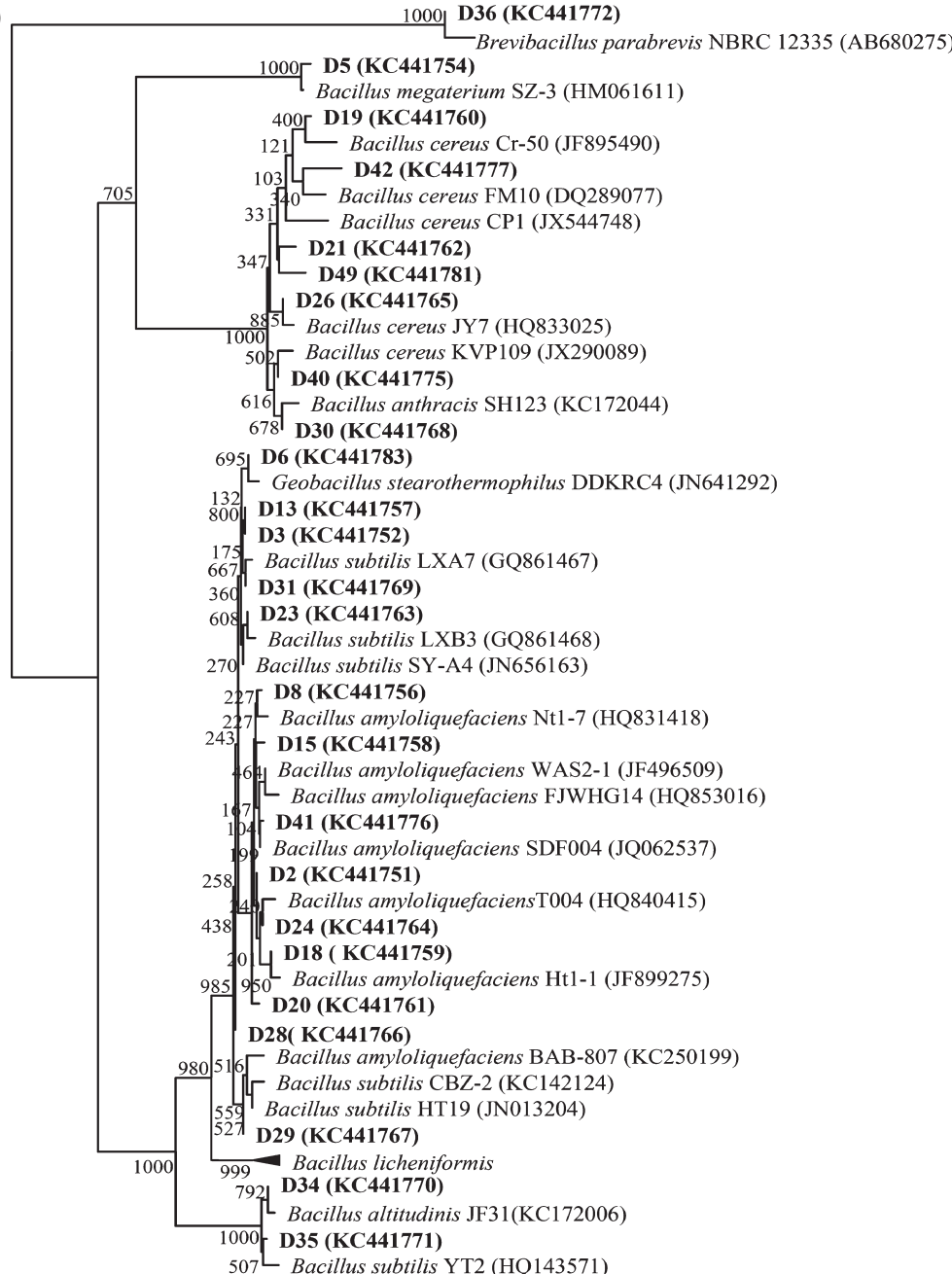

(b)

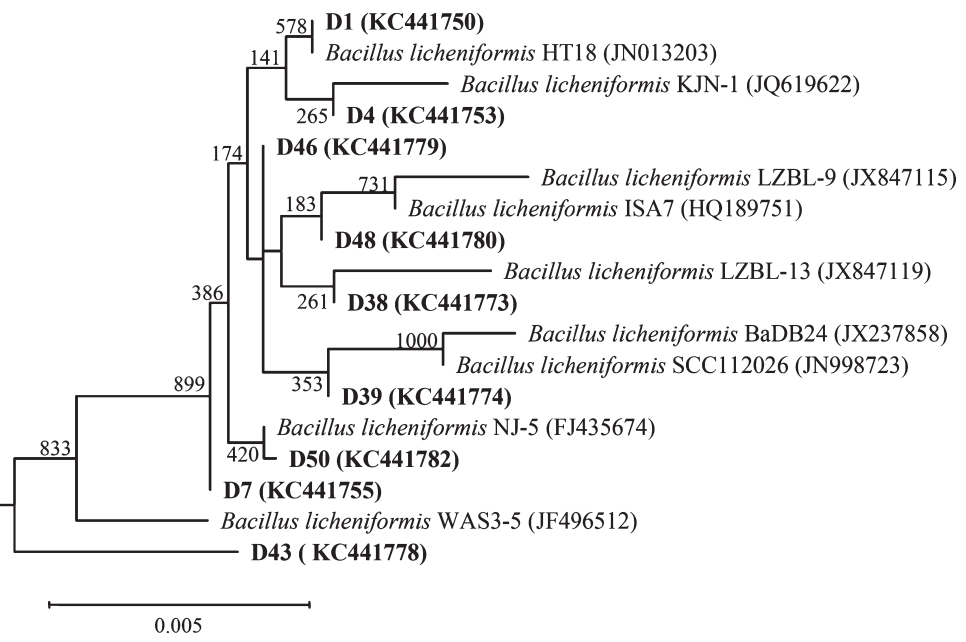

Figure 1 Phylogenetic relationships between bacterial 16S rDNA sequences in Deshan Daqu and previously reported sequences (a: main tree, b: subtree of Bacillus licheniformis). The number on each branch indicates the percentage of 1000 replicates that included the branch. Sequences determined in this study are shown in bold, and GenBank accession numbers are shown in parentheses for all of the related sequences. The scale bar of 0.02 (a) and 0.005 (b) represents a $2 \%$ and $5 \%$ nucleotide substitution rates according to the Jukes-Cantor evolutionary distance, respectively. 
Figure 2 Phylogenetic relationships between bacterial 16S rDNA sequences in Baisha Daqu and previously reported sequences. The number on each branch indicates the percentage of 1000 replicates that included the branch. Sequences determined in this study are shown in bold, and GenBank accession numbers are shown in parentheses for all of the related sequences. The scale bar of 0.02 represents a $2 \%$ nucleotide substitution rate according to the Jukes-Cantor evolutionary distance.

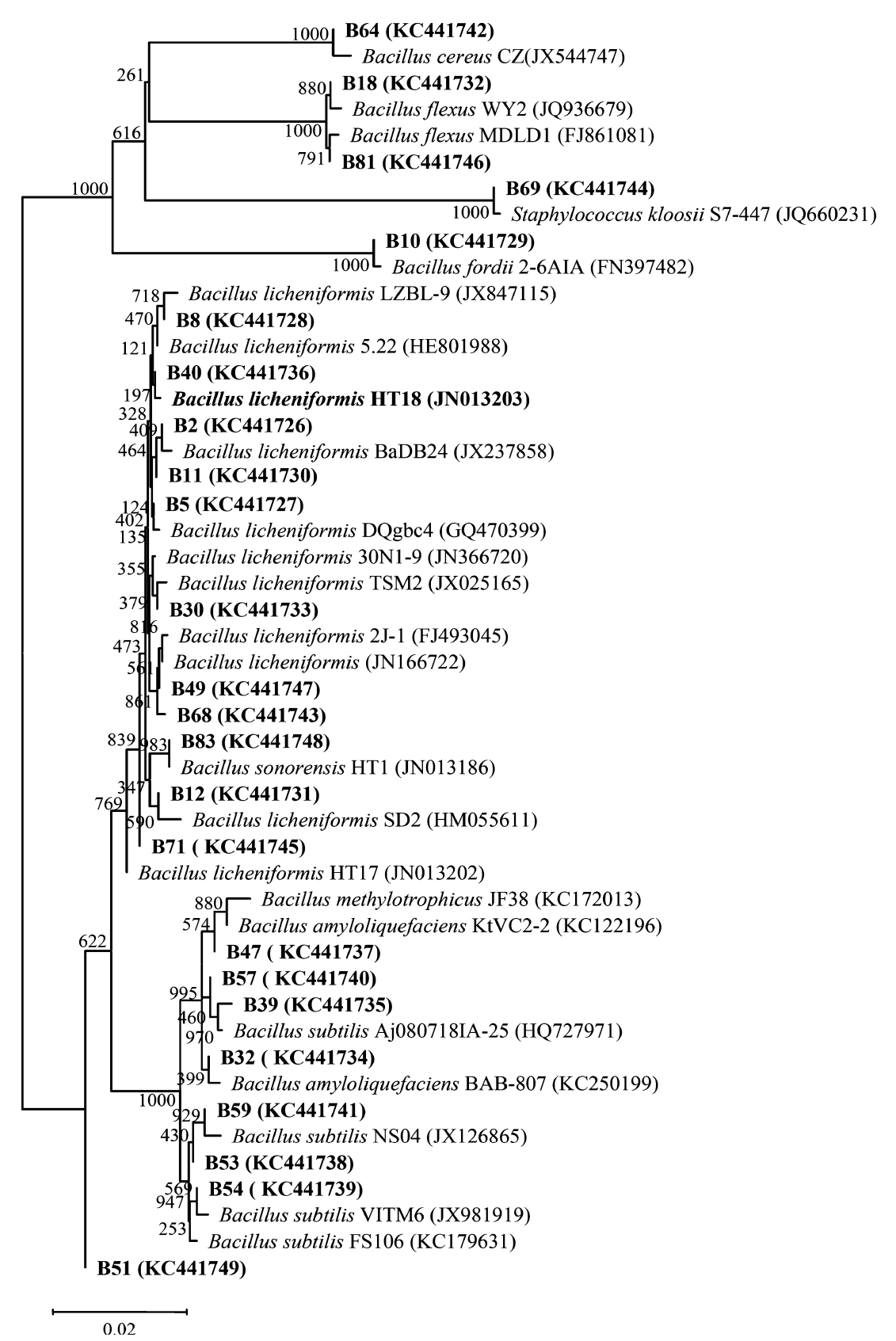

To determine diversity in phenotypic functions, extracellular amylase screening assay was conducted among all fifty-six bacterial isolates from Wuling Daqu. A total of sixteen and ten isolates demonstrated $\alpha$-amylase and glucoamylase production, respectively (Table 4 ). The maximum $\alpha$-amylase yield of $22.1 \mathrm{U} \mathrm{mL}^{-1}$ was obtained from $B$. licheniformis W61, whereas B. amyloliquefaciens $\mathrm{W} 25$ produced the maximum glucoamylase yield of $30.3 \mathrm{U} \mathrm{mL}^{-1}$. The activity of $\alpha$-amylase and glucoamylase produced by
B. licheniformis, B. subtilis, B. amyloliquefaciens and B. oleronius accounted for $33.5 \%, 12.9 \%, 27.4 \%$ and $22.6 \%$, and $20.0 \%, 39.8 \%, 19.1 \%$ and $24.7 \%$, respectively. However, B. circulans and B. sonorensis could produce neither $\alpha$-amylase nor glucoamylase.

The variety of bacteria found in the Daqus produced complicated enzyme systems that converted the Daqus into unique glycation and fermented substances, which are useful for subsequent reactions leading to flavour production (Shi et al., 2009). The variety of bacteria 


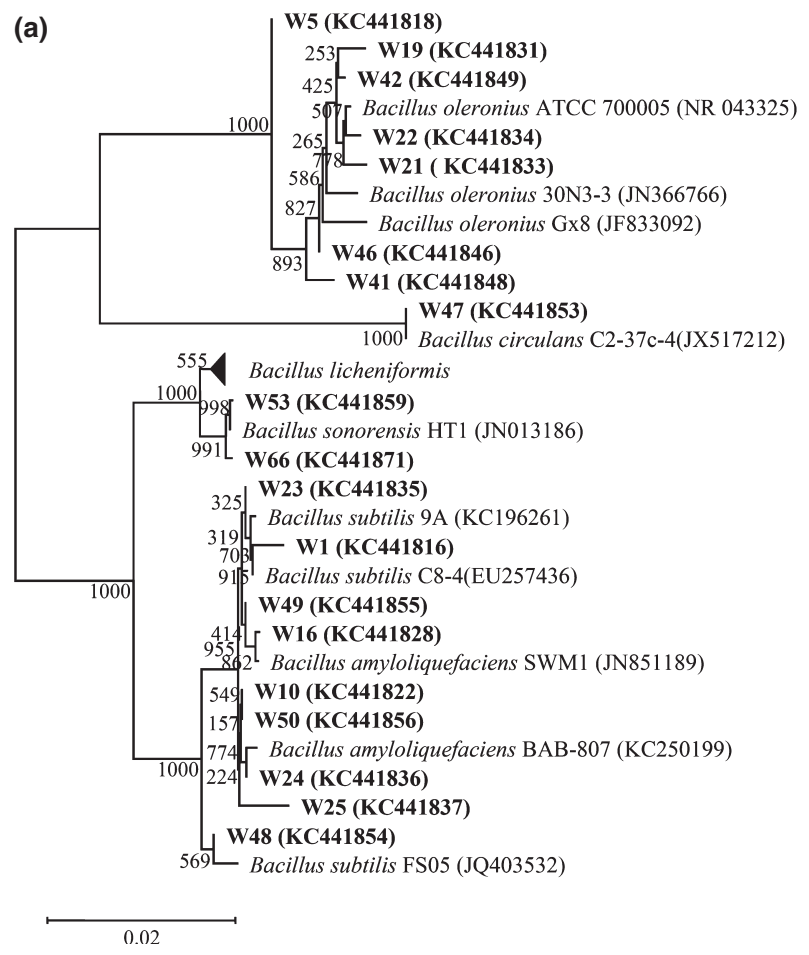

Figure 3 Phylogenetic relationships between bacterial 16S rDNA sequences in Wuling Daqu and previously reported sequences (a: main tree, b: subtree of Bacillus licheniformis). The number on each branch indicates the percentage of 1000 replicates that included the branch. Sequences determined in this study are shown in bold, and GenBank accession numbers are shown in parentheses for all of the related sequences. The scale bar of 0.02 (a) and 0.002 (b) represents a $2 \%$ nucleotide substitution rate according to the Jukes-Cantor evolutionary distance, respectively.

observed in the three Daqus could produce amylase, but the compositions of bacteria for producing amylase were obviously different, although they were produced in Hunan province (Tables 2-4). As the dominant bacterial species between the three Daqus, B. licheniformis and $B$. subtilis also possessed high activities of $\alpha$-amylase and glucoamylase that enabled them to utilise raw starch as a carbon source, which is a very important nutrient during fermentation (Tables 2-4). Bacillus licheniformis and B. subtilis may facilitate the conversion of starch into fermentable carbohydrates due to amylolytic activity, which may generate suitable substrates for the second stage of spirit production, that is, the alcoholic fermentation (Zheng et al., 2012). Bacillus amyloliquefaciens was one of the dominant bacterial species producing amylase between Deshan, Baisha and Wuling Daqus. Bacillus amyloliquefaciens, which is one of the most widely used species for the bulk production of amylase, is a potent producer of amylase and other industrial exoenzymes (Tanyildizi et al., 2007; Liu et al., 2012). Moreover, B. cereus was also one of the main bacterial species producing amylase in Deshan Daqu. It was reported that amylase was produced by $83 \%$ and $46 \%$ of B. cereus strains isolated from cheese in Turkey and legume-based Indian fermented foods (Roy et al., 2007; Molva et al., 2009). Bacillus oleronius was also another predominant bacterial species producing amylase in Wuling Daqu, which was also detected in Maotai Daqu (Liu et al., 2012). However, other bacterial species, for example, B. anthracis, could produce both $\alpha$-amylase and glucoamylase merely in Deshan Daqu. By comparison, B. flexus could secrete both $\alpha$-amylase and glucoamylase only in Baisha Daqu. Additionally, no other bacterial species could produce either $\alpha$-amylase or glucoamylase in Wuling Daqu. It was observed that different bacterial species could produce different yields of $\alpha$-amylase and glucoamylase between the three Daqus. The maximum $\alpha$-amylase yields produced by $B$. amyloliquefaciens D15 and B. subtilis B39 are $63.8 \%$ and $19.9 \%$ higher than that by $B$. licheniformis W61. Meanwhile, the maximum glucoamylase yield secreted by $B$. licheniformis D38 and B. subtilis B53 is 2.1-fold and 1.7-fold higher than that by $B$. amyloliquefaciens W25.

The bacterial diversity in the three Daqus was closely related to the types of spirits. Moreover, the bacterial diversity in the Daqu was affected by the environmental condition, production techniques, raw materials and storage time. Many intrinsic and extrinsic factors of Daqu production influence the richness and structure of the bacterial community including raw material variety, moisture content, temperature control, geographical location, climate and water (Wang et al., 2011). Zhang et al. (2013) studied the potential of Bacillus licheniformis as a starter culture for aroma concentration improvement in the fermentation of Chinese Moutai-flavour liquor. Yan et al. (2013) reported that B. licheniformis and B. subtilis can influence the organic acids in Daqu significantly and differently. The three typical Daqus related to flavour production should be studied in the future to establish the connection between the cultivable bacterial diversity, amylase production and the flavour intensity or the volatile compounds.

\section{Conclusion}

This study is the first application using culture-dependent method and 16S rRNA molecular technique to simultaneously investigate the cultivable bacterial diversity and amylase production in the three typical Daqus of Deshan, Baisha and Wuling spirits. The results showed that the indigenous bacterial communities in the three Daqus were complex and consisted of a wide variety of bacteria. Both cultivable bacterial diversity and amylase production were obviously different. Each Daqu contained some unique bacteria 
(b)

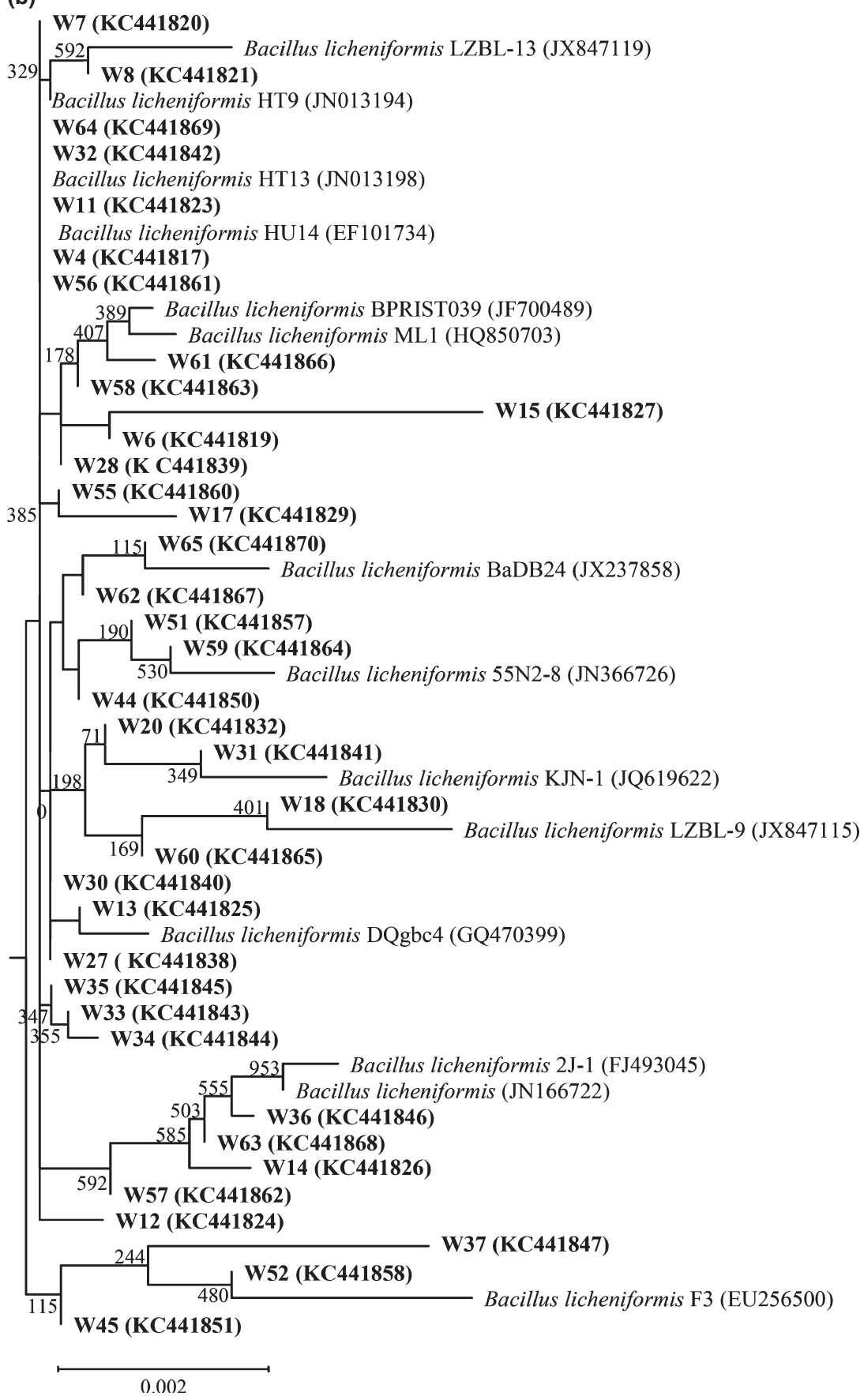

Figure 3 (Continued)

enables them to utilise raw starch as a carbon source. The diversity of bacterial species and metabolisms might be linked to the flavour intensity. Further investigation needs to be carried out for obtaining more detailed information on the specific function of each individual microorganism and its contribution to the final formation of the unique aroma in Chinese spirits. 


\section{Acknowledgments}

The authors are thankful to those who provided the Daqu samples for this research. This research was supported by the National Key Technology R\&D Program of China (No. 2012BAK17B11) and Beijing Municipal Commission of Education and Subsidy for Outstanding People of Beijing (No. KM201311417007; PXM2013_014209_07_000082; 2011A005022000004).

\section{References}

Chen, T.T., Wang, M.J., Jiang, S.Y., Xiong, S.Q., Zhu, D.C. \& Wei, H. (2011). Investigation of the microbial changes during kojimaking process of Douchi by culture-dependent techniques and PCR-DGGE. International Journal of Food Science and Technology, 46, 1878-1883.

Dhale, M.A. \& Vijay-Raj, A.S. (2009). Pigment and amylase production in Penicillium sp NIOM-02 and its radical scavenging activity. International Journal of Food Science and Technology, 44, 2424-2430.

Gupta, S., Rajauria, G. \& Abu-Ghannam, N. (2010). Study of the microbial diversity and antimicrobial properties of Irish edible brown seaweeds. International Journal of Food Science and Technology, 45, 482-489.

Hayes, M.M., Hughes, T.A. \& Greene, A.K. (2012). Bacterial diversity in dried colostrum and whey sold as nutraceutical products. Journal of Food Science, 77, M359-M363.

Huang, J.J., Wang, S.Y., Zhang, L.Y. et al. (2012). Isolation and identification of high pressure-resistant bacteria naturally contaminating strawberry pulp. International Journal of Food Science and Technology, 47, 2620-2626.

Kumar, P. \& Satyanarayana, T. (2009). Overproduction of glucoamylase by a deregulated mutant of a thermophilic Mould thermomucor indicae-seudaticae. Applied Biochemistry and Biotechnology, 158, 113-125.

Li, Z.M., Bai, Z.H., Zhang, B.G. et al. (2005). Newly isolated Bacillus gibsonii S-2 capable of using sugar beet pulp for alkaline pectinase production. World Journal of Microbiology and Biotechnology, 21, 1483-1486.

Li, X.R., Ma, E.B., Yan, L.Z. et al. (2011). Bacterial and fungal diversity in the traditional Chinese liquor fermentation process. International Journal of Food Microbiology, 146, 31-37.

Li, Z.M., Bai, Z.H., Zhang, B.G. et al. (2012). Purification and characterization of alkaline pectin lyase from a newly isolated Bacillus clausii and its application in elicitation of plant disease resistance. Applied Biochemistry and Biotechnology, 167, 2241-2256.

Liu, X., Guo, K.L. \& Zhang, H.X. (2012). Determination of microbial diversity in Daqu, a fermentation starter culture of Maotai liquor, using nested PCR-denaturing gradient gel electrophoresis. World Journal of Microbiology and Biotechnology, 28, 2375-2381.

Minh, H.K.Q. \& Rakshit, S.K. (2009). Use of specific PCR-based molecular markers for discrimination, rapid analysis of purity and identification of six fragrant rice. International Journal of Food Science and Technology, 44, 1959-1965.

Molva, C., Sudagidan, M. \& Okuklu, B. (2009). Extracellular enzyme production and enterotoxigenic gene profiles of Bacillus cereus and Bacillus thuringiensis strains isolated from cheese in Turkey. Food Control, 20, 829-834.

Qian, J.Q., Chen, X.Y., Ying, X.X. \& Lv, B.F. (2011). Optimisation of porous starch preparation by ultrasonic pretreatment followed by enzymatic hydrolysis. International Journal of Food Science and Technology, 46, 179-185.

Qin, H., Huo, D.Q., Zhang, L. et al. (2012). Colorimetric artificial nose for identification of Chinese liquor with different geographic origins. Food Research International, 45, 45-51.

Roy, A., Moktan, B. \& Sarkar, P.K. (2007). Characteristics of Bacillus cereus isolates from legume-based Indian fermented foods. Food Control, 18, 1555-1564.

Shankar, M., Priyadharshini, R. \& Gunasekaran, P. (2009). Quantitative digital image analysis of chromogenic assays for high throughput screening of $\alpha$-amylase mutant libraries. Biotechnology Letters, 31, 1197-1201.

Shi, J.H., Xiao, Y.P., Li, X.R., Ma, E.B., Du, X.W. \& Quan, Z.X. (2009). Analyses of microbial consortia in the starter of Fen Liquor. Letters in Applied Microbiology, 48, 478-485.

Smith, M.R. \& Zahnley, J.C. (2005). Characteristics of the amylase of Arthrobacter psychrolactophilus. Journal of Industrial Microbiology \& Biotechnology, 32, 439-448.

Tanyildizi, M.S., O"zer, D. \& Elibol, M.. (2007). Production of bacterial $\alpha$-amylase by $B$. amyloliquefaciens under solid substrate fermentation. Biochemical Engineering Journal, 37, 294-297.

Wang, C.L., Shi, D.J. \& Gong, G.L. (2008). Microorganisms in Daqu: a starter culture of Chinese Maotai-flavor liquor. World Journal of Microbiology and Biotechnology, 24, 2183-2190.

Wang, H.Y., Gao, Y.B., Fan, Q.W. \& Xu, Y. (2011). Characterization and comparison of microbial community of different typical Chinese liquor Daqus by PCR-DGGE. Letters in Applied Microbiology, 53, 134-140.

Xie, Z., Zhang, Z.L., Hanzlik, S., Cook, E. \& Shen, Q.J. (2007). Salicylic acid inhibits gibberellins -induced alpha-amylase expression and seed germination via a pathway involving an abscisic-acid-inducible WRKY gene. Plant Molecular Biology, 64, 293303.

Yan, Z., Zheng, X.W., Chen, J.Y., Han, J.S. \& Han, B.Z. (2013). Effect of different Bacillus strains on the profile of organic acids in a liquid culture of Daqu. Journal of the Institute of Brewing, 119, 78-83.

Yoshizaki, Y., Susuki, T., Takamine, K., Tamaki, H., Ito, K. \& Sameshima, Y. (2010). Characterization of glucoamylase and $\alpha$-amylase from Monascus anka: enhanced production of $\alpha$-amylase in red koji. Journal of Bioscience and Bioengineering, 6, 670-674.

Zhang, C.L., Ao, Z.H., Chui, W.Q., Shen, C.H., Tao, W.Y. \& Zhang, S.Y. (2011). Characterization of volatile compounds from Daqu-a traditional Chinese liquor fermentation starter. International Journal of Food Science and Technology, 46, 1591-1599.

Zhang, R., Wu, Q. \& Xu, Y. (2013). Aroma characteristics of Moutai-flavour liquor produced with Bacillus licheniformis by solid-state fermentation. Letters in Applied Microbiology, 57, 11-18.

Zheng, X.W., Tabrizi, M.R., Nout, M.J.R. \& Han, B.Z. (2011). Daqu-a traditional Chinese liquor fermentation starter. Journal of the Institute of Brewing, 1, 82-90.

Zheng, X.W., Yan, Z., Han, B.Z. et al. (2012). Complex microbiota of a Chinese "Fen" liquor fermentation starter (Fen-Daqu), revealed by culture-dependent and culture-independent methods. Food Microbiology, 31, 293-300. 